\title{
Direct Tissue Analysis of Phospholipids in Rat Brain Using MALDI-TOFMS and MALDI-Ion Mobility-TOFMS
}

\author{
Shelley N. Jackson, Hay-Yan J. Wang, and Amina S. Woods \\ Intramural Research Program, National Institute on Drug Abuse, National Institutes of Health, \\ Baltimore, Maryland, USA
}

\author{
Michael Ugarov, Thomas Egan, and J. Albert Schultz \\ Ionwerks, Inc., Houston, Texas, USA
}

\begin{abstract}
After water, lipids are the most common biomolecules found in the brain (12\%). A brief perusal of the physiology, anatomy, and pathophysiology of the brain illustrates the importance of lipids. Recent advances in mass spectrometry have allowed the direct probing of tissues. However, most studies have focused on proteins. In the present work, matrix-assisted laser desorption/ionization time-of-flight mass spectrometry (MALDI-TOFMS) and MALDI-ion mobility (IM)-TOFMS were employed for direct analysis of phospholipids in rat brain tissue. Molecular ions $\left(\mathrm{MH}^{+}\right)$corresponding to phosphatidylcholines, phosphatidylethanolamines, and sphingomyelin, were recorded. When studying pharmacology, we learn that many therapeutic compounds are stored in the body's adipose tissue. MALDI-TOFMS and MALDIIM-TOFMS were thus used to analyze rat brain tissue with chlorisondamine added directly onto the tissue slice. With both techniques, noncovalent complexes between the tissue phospholipids and chlorisondamine were detected. In addition, MALDI-IM-TOFMS of noncovalent complexes between phospholipids and chlorisondamine displayed a mobility between that of an isobaric lipid and peptide. (J Am Soc Mass Spectrom 2005, 16, 133-138) (C) 2004 American Society for Mass Spectrometry
\end{abstract}

$\mathrm{P}$ hospholipids represent a major group of lipids, present in abundance in all biological membranes. The general structural requirement for phospholipids is the presence of an alcohol, phosphate, and a fatty acid chain. However, a quaternary amine is often present. Major classes of phospholipids include phosphatidylcholine, phosphatidylethanolamine, phosphatidylserine, and sphingomyelin. Lipids, in particular phospholipids, are a major component of the brain [1]. Altered levels of phospholipids in tissue are associated with Farber disease, Gaucher disease, NiemannPick disease, Alzheimer disease, and Down syndrome [2-5]. Furthermore, sphingomyelin and other glycolipids have been shown to form noncovalent complexes with chlorisondamine, a nicotinic antagonist [6, 7], as well as with acetylcholine and peptides containing two or more adjacent Arg residues [8]. Traditional methods for the detection of phospholipids in tissue by mass spectrometry involve extraction and possible purification prior to mass analysis [9]. These sample prepara-

Published online December 9, 2004

Address reprint requests to Dr. A. S. Woods, Intramural Research Program, National Institute on Drug Abuse, National Institutes of Health, 5500 Nathan Shock Drive, Baltimore, MD 21224, USA. E-mail: awoods@intra.nida.nih.gov tion steps are time consuming and have to be altered according to the specific phospholipid to be detected.

Currently, matrix-assisted laser desorption/ionization time-of-flight mass spectrometry (MALDI-TOFMS) is being developed for the direct analysis of large biomolecules, mainly peptides and proteins, from tissue [10-12]. In these studies, MALDI mass spectral profiles have been generated to map the location of biomolecules or drugs in tissue. MALDI is well-suited for the direct analysis of biomolecules in tissue because of its high sensitivity, high tolerance for salts and other contaminants, and a wide mass range with little fragmentation. Despite the success of MALDI for the direct analysis of peptides and proteins in tissue, very little work has been undertaken for the direct tissue analysis of lipids, probably because most of the lipids in tissue have a molecular weight below $1000 \mathrm{Da}$, which is a spectral region that is complicated by chemical noise and mass interferences from matrix molecular ions. In addition, identification of lipid analytes can be difficult because of background interference ions from the preparation of tissue sections, i.e., stains, optimal cutting temperature compound, etc. However, MALDI was recently used for the direct analysis of phospholipids in slices of fresh and fixed lens tissue [13]. In this work, 
two classes of phospholipids, phosphatidylcholines and sphingomyelins, were studied for regional analysis in tissue.

Ion mobility (IM) spectrometry is a robust method that allows for the rapid separation and analysis of a wide range of compounds [14]. In this technique, ions can be generated by several methods such as pyrolysis, electrospray, and laser desorption prior to entering a gas-filled mobility drift cell region. In the drift cell, ions obtain an average drift velocity from an electric field based upon their collision cross section $(\Omega)$ or shape, which allows for the separation of different shaped molecules [14, 15]. The coupling of IM to mass spectrometry (IM-MS) has been used for the analysis of a large variety of samples including explosives, drug molecules, biomolecules, etc. [16, 17]. Furthermore, IM-MS has been employed successfully for the analysis of structural isomers [18]. The combining of a MALDI source with ion-mobility has allowed for a wide range of samples to be analyzed by MALDI-IM $[19,20]$. In a recent study, MALDI-IM has been used to analyze complex mixtures of test biomolecules of known structure, including sphingomyelin and other lipids [7]. ${ }^{\circ}$ In that work, isobaric lipid, peptide, and oligonucleotides were preseparated prior to mass analysis by differences of up to $30 \%$ in ion mobility drift time.

In this work, we report on the direct analysis of phospholipids in rat brain tissue using conventional MALDI-TOFMS and MALDI- IM-TOFMS. Nine different molecular species of phospholipids, consisting of phosphatidylcholines, phosphatidylethanolamine, and sphingomyelin, were identified by determining both the parent ion $\mathrm{m} / \mathrm{z}$ and their ion mobility drift times, which are characteristic of lipid molecules. Direct analysis of tissue using MALDI-IM-TOFMS resulted in 2-D mass spectra with separate trend lines corresponding to lipids, peptides, and oligonucleotides. Brain tissue with chlorisondamine added was also analyzed by MALDITOFMS and MALDI- IM-TOFMS. In both cases, noncovalent complexes were observed between the tissue phospholipids and chlorisondamine. Furthermore, in MALDI- IM-TOFMS, these noncovalent complexes exhibited a mobility between that of an isobaric lipid and peptide.

\section{Experimental}

\section{Mass Spectrometers}

A Voyager DE-Pro MALDI time-of-flight instrument (PE-Biosystems, Framingham, MA) was used in this study for conventional MALDI analysis. A nitrogen laser $(337 \mathrm{~nm})$ at a repetition rate of $3 \mathrm{~Hz}$ was employed for ionization. Samples were analyzed in both linear and reflectron mode with delayed extraction at an accelerating voltage of $20 \mathrm{kV}$. Mass spectra acquired in linear mode were the sum of 25 laser shots, while mass spectra acquired in reflectron mode were the sum of 100 laser shots. All spectra were acquired in positive ion mode.

MALDI-IM-TOFMS data were acquired using a prototype periodic focusing ion mobility instrument (Ionwerks Inc., Houston, TX). A mobility resolution of 30 (FWHM of mobility peak/drift time) and a mass resolution of 2000 for $\mathrm{m} / \mathrm{z} 1000$ using an orthogonal timeof-flight mass spectrometer (o-TOFMS) are routinely achieved. The length of the drift cell is $15 \mathrm{~cm}$ and is operated at $\sim 1600 \mathrm{~V}$ with 5 torr helium pressure. A Nd-YLF high repetition rate pulsed UV laser (349 nm, up to $1 \mathrm{kHz}$ ) is used to generate ions. The ions drift to the end of the mobility cell under the force of a high voltage field applied between the sample plate and successive electrode rings within the mobility spectrometer. The ions then exit the mobility cell through a differentially pumped skimmer region into the mass spectrometer for analysis. The mobility drift times are typically up to $1 \mathrm{~ms}$, while the flight times within the mass spectrometer are $25 \mu \mathrm{s}$ or less. Therefore, more than 400 interleaved mass spectra can be obtained after every laser pulse depending on the target mass range. This process is repeated for several hundred laser shots, until the data contain sufficient intensity to permit analysis. In this work, the data is presented as 2-D contour plots of ion intensity as a function of ion mobility drift time (y-axis) and $m / z$ (x-axis). Furthermore, for each MALDI-IM plot, the derived 1-D ion mobility spectrum and 1-D mass spectrum are also included. In the 2-D contour plots of ion mobility versus mass, compounds that have the same molecular weight but different structures are observed along ion groupings, which have different slopes. In order to highlight these ion groupings, "trend lines" were added by visual inspection. All contour plots were produced using IDL software (Research Systems, Boulder, CO).

\section{Tissue Sectioning}

The use and handling of animals in this study abide by the Guide for the Care and Use of Laboratory Animals (NIH). Male Spray-Dawley rats (Harlan Industries, Indianapolis, IN) between 300 and $420 \mathrm{~g}$ were used. Rats were euthanized with sodium pentobarbital intraperitoneal injection $(>65 \mathrm{mg} / \mathrm{kg})$ and were decapitated upon cessation of respiration. The brains were quickly removed from the skull and frozen in dry ice-chilled isopentane for $15 \mathrm{~s}$, prior to storage at $-80^{\circ} \mathrm{C}$.

Frozen brain tissue was cut into thin sections $(14 \mu \mathrm{m}$ thickness) in a cryostat (CM 3050 S, Leica Microsystems Nussloch GmbH, Nussloch, Germany). The tissue samples were attached to the cryostat sample stages using ice slush made from distilled water. The ice slush only came in contact with the tissue blocks at the surface opposing the sample stages, and was frozen into a thin layer of ice within $5 \mathrm{~s}$. It was decided to use water instead of optimal cutting temperature compound (OCT) to connect the tissue to the sample stage, because past studies showed that OCT interference can reduce 
the ${ }^{\circ}$ quality ${ }^{\circ}$ f $^{\text {the }}{ }^{\circ}$ mass ${ }^{\circ}$ spectra $[10]$. Serial'brain ${ }^{\circ}$ sections were alternately collected onto a MALDI sample target and poly-L-lysine coated microscopic slide. After collection, tissue sections were stored at $4{ }^{\circ} \mathrm{C}$ until MALDI analysis. The tissue sections on the microscopic slides were stained with cresyl violet and a Rat Brain Atlas $[21]^{\circ}$ was $^{\circ}$ used $^{\circ}$ for $^{\circ}$ the $^{\circ}$ assignment $^{\circ}$ of ${ }^{\circ}$ the ${ }^{\circ}$ brain ${ }^{\circ}$ regions analyzed by mass spectrometry.

\section{Chemicals}

Chlorisondamine iodide was acquired from Tocris (St. Louis, MO) and was diluted in water to a concentration of $1 \mathrm{nmol} / \mu \mathrm{L}$. The following MALDI matrices were used in this study: 6-aza-2-thiothymine (Aldrich, Milwaukee, WI), 2,6-dihydroxyacetophenone (Fluka, Buchs, Switzerland), $\alpha$-cyano-4-hydroxy cinnamic acid (Aldrich), and sinapic acid (Fluka). All matrices were initially prepared as a saturated solution in 50\% ethanol. Upon further testing, a $1: 1$ (vol/vol) dilution of 6-aza-2-thiothymine or a 1:4 and $1: 9$ (vol/vol) dilution of 2,6-dihydroxyacetophenone in $50 \%$ ethanol were shown to yield optimal mass spectral results.

\section{Sample Preparation}

Matrix solution volumes of $0.1,0.5$, and $1 \mu \mathrm{L}$ were deposited directly on the tissue section and allowed to air-dry prior to insertion into the mass spectrometer. For tissue sections with chlorisondamine, $1,0.5$, or $0.1 \mu \mathrm{L}$ of the chlorisondamine solution was deposited onto the tissue and allowed to air-dry. Next, matrix solution was deposited onto the tissue section and allowed to air-dry prior to mass analysis.

\section{Results and Discussions}

Initial work was conducted to determine the optimal matrix and matrix concentration for analysis of phospholipids in tissue. Four common MALDI matrices, 6-aza-2thiothymine (ATT), 2,6-dihydroxyacetophenone (DHA), $\alpha$-cyano-4-hydroxy cinnamic acid (CHCA), and sinapic acid (SA) were tested. From these experiments, it was determined that a 1:1 ( $\mathrm{vol} / \mathrm{vol})$ dilution of saturated ATT solution and a 1:4 and 1:9 (vol/vol) dilution of saturated DHA solution in 50\% ethanol produced the best quality (signal:noise, low matrix interference peaks, resolution) mass spectra. Phospholipids were observed using CHCA and SA, but the quality of the mass spectra and the ability to identify phospholipid species was difficult due to matrix interference peaks.

Figure $1^{\circ}$ illustrates ${ }^{\circ} a^{\circ}$ typical $^{\circ} \mathrm{MALDI}^{\circ}$ mass $^{\circ}$ spectrum ${ }^{\circ}$ of rat brain tissue in the mass range of the phospholipids molecular species. This mass spectrum was obtained from the cerebral cortex region of the brain in reflectron mode with DHA matrix. Three different classes of phospholipids: phosphatidylcholines (PC), phosphatidylethanolamines (PE), and sphingomyelins (SM), were identified by ${ }^{\circ}$ direct ${ }^{\circ}$ issue ${ }^{\circ}$ analysis ${ }^{\circ}$ ising ${ }^{\circ}$ MLDI. Table 1 Tists $^{\circ}$ all the

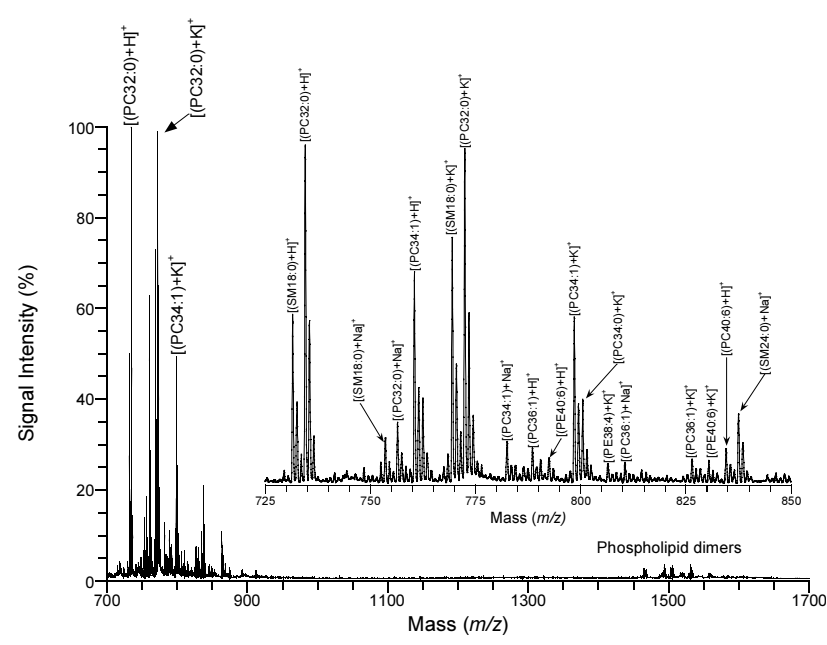

Figure 1. MALDI mass spectrum of the cerebral cortex region with DHA matrix in reflectron mode.

phospholipid species identified. The most dominant mass peaks ${ }^{\circ}$ in ${ }^{\circ}$ Figure ${ }^{\circ} 1^{\circ}$ correspond $^{\circ}$ to ${ }^{\circ}$ the ${ }^{\circ}$ following ${ }^{\circ}$ molecular species: PC 32:0, PC 34:1, and SM 18:0. The molecular ions, $[\mathrm{M}+\mathrm{H}]^{+},[\mathrm{M}+\mathrm{Na}]^{+}$, and $[\mathrm{M}+\mathrm{K}]^{+}$, were observed for all three species. The domination of the mass spectrum by peaks associated with PCs and SMs is expected, since these two classes of phospholipids account for more than $50 \%$ of membrane phospholipids in eukariotic organisms [22].$^{\circ}$ Furthermore, ${ }^{\circ}$ the ${ }^{\circ}$ presence ${ }^{\circ}$ of ${ }^{\circ} a^{\circ}$ positively ${ }^{\circ}$ charged quaternary ammonium group in both phosphatidycholines and sphingomyelins aid in the detection of these two classe ${ }^{\circ}$ of $^{\circ}$ phospholipids $^{\circ}$ in $^{\circ}$ positive ${ }^{\circ}$ ion $^{\circ}$ mode. ${ }^{\circ}$ Previous MALDI-MS studies of phospolipid mixtures have demonstrated that in positive ion mode phosphatidylcholine and sphingomyelin species can inhibit the detection of other

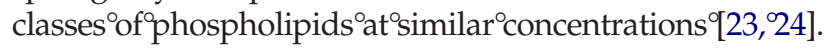
Several additional regions of the rat brain, including the hippocampus and hypothalamus, were analyzed by MALDI-MS. Mass spectral profiles of phospholipids were similar for the brain regions that were tested. PC 32:0, PC 34:1, and SM 18:0 were the dominant phospholipid species detected in all of the brain regions that were analyzed.

Table 1. Phospholipid molecular species detected in brain tissue

\begin{tabular}{cc}
\hline Species $^{\text {a }}$ & Protonated molecule (Da) \\
\hline \hline PC 32:0 & 734.6 \\
PC 34:0 & 762.6 \\
PC 34:1 & 760.6 \\
PC 36:1 & 788.6 \\
PC 40:6 & 834.6 \\
PE 38:4 & 768.6 \\
PE 40:6 & 792.6 \\
SM 18:0 & 731.6 \\
SM 24:0 & 815.7 \\
\hline
\end{tabular}

aPhosphatidylcholine (PC) and phosphatidylethanolamine (PE) species number equal the total length and number of double bonds of both acyl chains. Sphingomyelin (SM) species number corresponds to the length and number of double bonds of the acyl chain attached to the sphingosine base. 


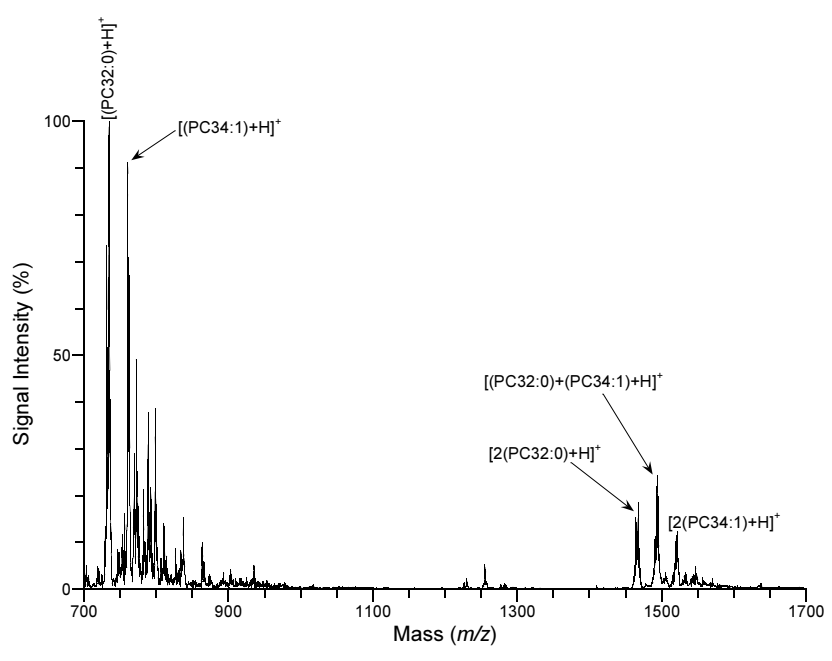

Figure 2. MALDI mass spectrum of the cerebral cortex region with ATT matrix in linear mode.

Figure ${ }^{\circ}{ }^{\circ}$ hows ${ }^{\circ} a^{\circ}$ mass ${ }^{\circ}$ spectrum ${ }^{\circ}$ of ${ }^{\circ}$ the ${ }^{\circ}$ cerebral ${ }^{\circ}$ cortex region in ${ }^{\circ}$ linear ${ }^{\circ}$ mode ${ }^{\circ} w$ ith $^{\circ}{ }^{\circ} \mathrm{ATT}^{\circ}$ matrix. ${ }^{\circ}$ Similar to ${ }^{\circ}$ Figure 1, the major'peaks'in the mass'spectrum ${ }^{\circ}$ correspond ${ }^{\circ}$ to $\mathrm{PC}$ 32:0, PC 34:1, and SM 18:0. Note the phospholipid dimers seen around $1500 \mathrm{Da}$. Less intense phospholipid dimer signals ${ }^{\circ}$ were ${ }^{\circ}$ also ${ }^{\circ}$ observed $^{\circ}{ }^{\circ}{ }^{\circ}$ reflectron ${ }^{\circ}$ mode $^{\circ}$ (see ${ }^{\circ}$ Figure 1).The dimerization of 9 phospholipids has previously been reported for electrospray ionization mass spectrometry [25], ${ }^{\circ}$ liquid $^{\circ}$ secondary $^{\circ}$ ion $^{\circ}$ mass $^{\circ}$ spectrometry ${ }^{\circ}$ [26], MALDI-TOFMS $^{\circ}[8]^{\circ}$ and $^{\circ}$ MALDI-IM-TOFMS ${ }^{\circ}[7]^{\circ}{ }^{\circ}$ The three ${ }^{\circ}$ major $^{\circ}$ dimer $^{\circ}$ mass $^{\circ}$ peaks ${ }^{\circ}$ observed $^{\circ}$ in $^{\circ}$ Figure $^{\circ} 2$ corresponded to the phospholipid species of PC 32:0 and $\mathrm{PC}^{\circ}$ 34:1..$^{\circ}$ Although ${ }^{\circ}$ not $^{\circ}$ labeled $^{\circ}$ on $^{\circ}$ Figure $^{\circ} 2$, , dimer $^{\circ}$ salt adducts and dimers consisting of SM 18:0 were also detected.

Chlorisondamine, a nicotinic antagonist, consists of two quaternary amine groups and a tetrachloroisoindoline ring. It has a hydrophobic head and a hydrophilic body and tail. Previous MALDI mass spectrometry studies have demonstrated that chlorisondamine forms noncovalent ${ }^{\circ}$ complexes $^{\circ}$ with $^{\circ}$ sphingomyelin $^{\circ}\left[6,{ }^{\circ} 7\right] .^{\circ}$ In these studies, chlorisondamine and sphingomyelin were mixed in solution prior to mass analysis. These complexes consisted of one molecule of chlorisondamine added to sphingomyelin. Additionally, ab initio calculations of chlorisondamine suggest that the positively charged quaternary ammonium of chlorisondamine interacts with the phosphate group in sphingomyelin ${ }^{\circ}[6] .{ }^{\circ}$ Figure ${ }^{\circ}{ }^{\circ}$ shows $^{\circ} a^{\circ} \mathrm{MALDI}^{\circ} \mathrm{mass}^{\circ}$ spectrum $^{\circ}$ of ${ }^{\circ}$ rat brain tissue with $1 \mathrm{nmol}$ of chlorisondamine added. This mass spectrum was recorded in linear mode with DHA matrix. Noncovalent complexes between chlorisondamine and phosphatidylcholine molecular species PC32:0 and PC34:1 in the tissue are observed. Both PC32:0 and PC34:1 form complexes with the addition of one molecule of chlorisondamine. Additionally, complexes consisting of the addition of one molecule of chlorisondamine with the loss of $\left(\mathrm{CH}_{3}\right)_{3} \mathrm{~N}, 59 \mathrm{Da}$, are also detected. Further experiments were conducted

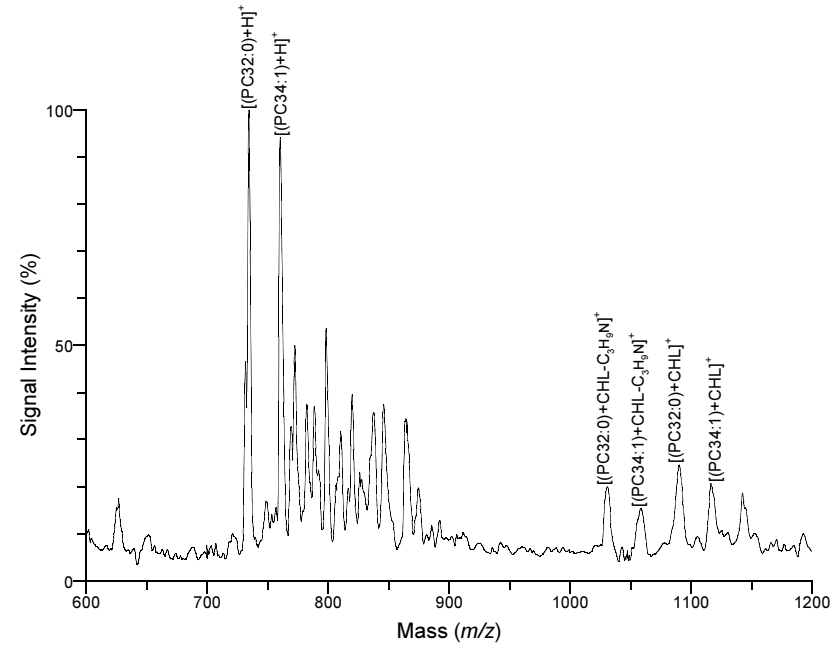

Figure 3. MALDI mass spectrum of brain tissue with $1 \mathrm{nmol}$ of chlorisondamine (CHL) added using DHA matrix in linear mode.

using ATT, CHCA, and SA matrices. However, only ATT $^{\circ}$ yielded $^{\circ}$ similar $^{\circ}$ results $^{\circ}$ as $^{\circ}$ seen $^{\circ}{ }^{\circ}{ }^{\circ}$ Figure $^{\circ} 3 .{ }^{\circ}$ This $^{\circ}$ is in agreement with previous studies of noncovalent complexes by MALDI in which complex formation was not observed when highly acidic matrices such as $\mathrm{CHCA}^{\circ}$ were $^{\circ}$ used $^{\circ}[27] .{ }^{\circ}$ Noncovalent $^{\circ}$ complexes $^{\circ}$ were also observed when 500 and 100 pmol of chlorisondamine were added to tissue sections (data not shown).

MALDI-IM-TOFMS was conducted on rat brain tissue slices onto which chlorisondamine solution was added. As previously demonstrated using test molecules of known structure, structurally different biomolecular ions, such as lipids, oligonucleotides and peptides form distinct familial trend lines on a 2-D plot of

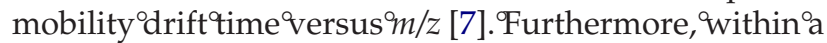
family of molecules, e.g., peptides, small (few percent) mobility drift time deviations are observed from a familial ${ }^{\circ}$ trend ${ }^{\circ} l i n e^{\circ}[28]^{\circ}{ }^{\circ}$ Comparable ${ }^{\circ}$ studies ${ }^{\circ}$ with ${ }^{\circ}$ lipids are $^{\circ}$ less $^{\circ}$ well $^{\circ}$ developed $[7] ;^{\circ}$ however, ${ }^{\circ}$ the ${ }^{\circ}$ mobility $^{\circ}$ of lipid ion species is consistently around $12 \%$ slower than isobaric peptides. MALDI-IM-TOFMS of rat brain tissue section with the addition of $1 \mathrm{nmol}$ chlorisondamine resulted in mobility-mass spectra with similarly wellseparated ${ }^{\circ}$ trend ${ }^{\circ}$ lines ${ }^{\circ}$ as ${ }^{\circ}$ illustrated ${ }^{\circ}{ }^{\circ}{ }^{\circ}$ Figure $^{\circ} 4 a^{\circ}$ and ${ }^{\circ} b$. These lines are not quantitative fits, but are rather added to guide the eye between major ions within a molecular class. More detailed studies to define a lipid familial trend line await an extension of our previous calibration ${ }^{\circ}$ studies ${ }^{\circ}[7]^{\circ}$ to $^{\circ}$ include ${ }^{\circ}$ more $^{\circ}$ compounds $^{\circ}$ of known structure. DHA matrix was used for this sample and, similar to MALDI-TOFMS results above, the dominant phospholipid species identified are PC 32:0, PC 34:1, and $^{\circ} \mathrm{SM}^{\circ}$ 18:0..$^{\circ}$ The $^{\circ}$ top $^{\circ}$ trend $^{\circ}$ line $^{\circ}$ in $^{\circ}$ Figure $^{\circ} 4 \mathrm{a}$ corresponds to lipids and the phospholipids are clearly distinguishable, while the bottom trend line corresponds to peptides and fragments. Furthermore, complexes of phospholipids with matrix, with chlorisondamine, and with matrix and chlorisondamine are 

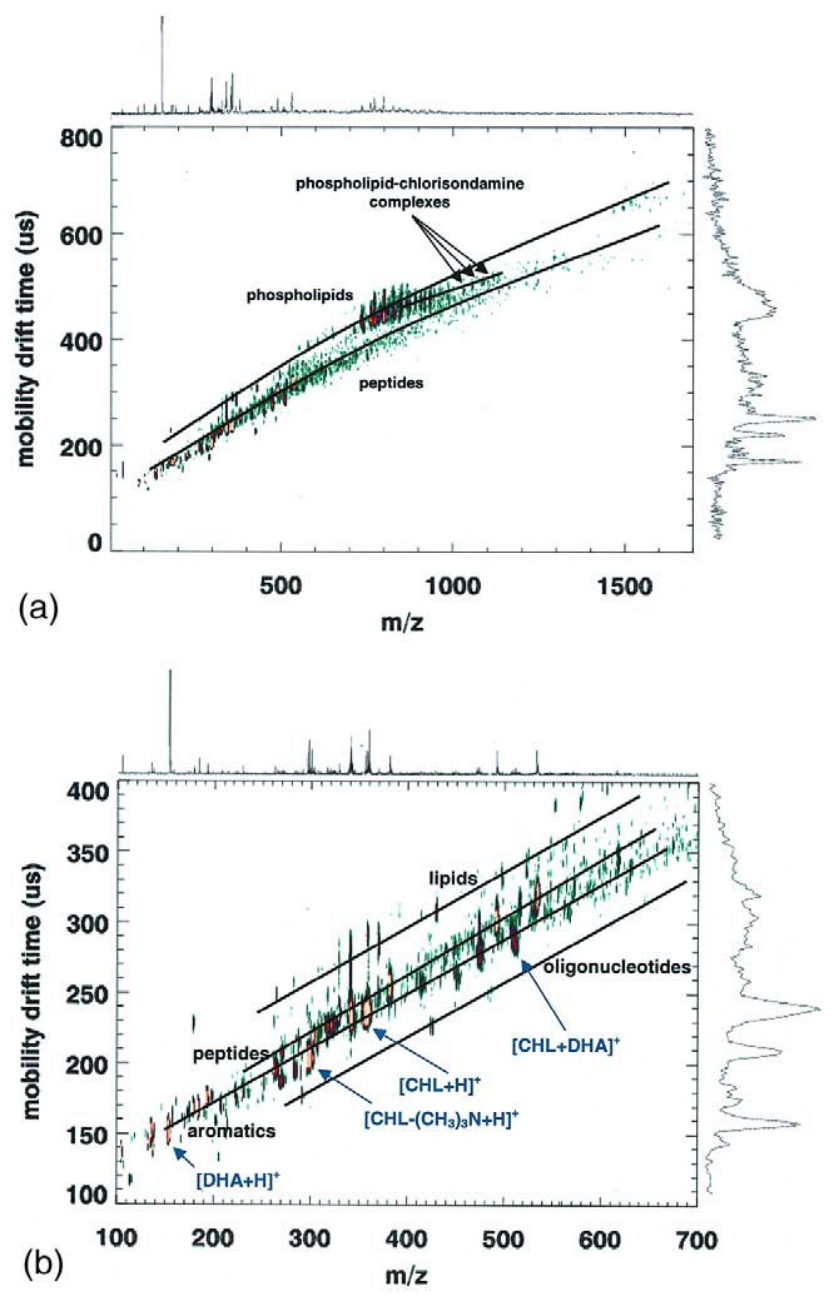

Figure 4. (a) MALDI-IM 2-D plot of brain tissue with $1 \mathrm{nmol}$ of chlorisondamine added using DHA matrix. (b) close-up of MALDI-IM 2-D plot from (a) below $700 \mathrm{Da}$.

observed and assigned in analogy to such complexes previously described using known test molecules of sphingomyelin [7]. In ${ }^{\circ}$ this ${ }^{\circ}$ previous ${ }^{\circ}$ work, ${ }^{9}{ }^{\circ}{ }^{\circ}$ was ${ }^{\circ}$ shown that such complexes deviate along a separate trend line located between the peptide and lipid trend lines as shown ${ }^{\circ}{ }^{\circ}$ Figure $^{\circ} 4$ a.

A more detailed view for the mass range below $700 \mathrm{Da}$ is $^{\circ}$ shown $^{\circ}$ in $^{\circ}$ Figure $^{\circ} 4 \mathrm{~b}^{\circ}{ }^{\circ}$ Based $^{\circ}$ on $^{\circ}$ our $^{\circ}$ earlier $^{\circ}$ results $^{\circ}[7]$, trend lines corresponding to lipids, peptides, and oligonucleotides can be identified. In this more restricted mass region, the trend lines can be approximated by straight lines '28] and 'we haveadded four'ines to guidethe eye in Figure $4 \mathrm{~b}$. ${ }^{\circ}$ In $^{\circ}$ addition ${ }^{\circ}$ to ${ }^{\circ}$ the ${ }^{\circ}$ lipids ${ }^{\circ}$ and ${ }^{\circ}$ peptides, ${ }^{\circ}$ one ${ }^{\circ}$ can see that ions peaks which are ascribed to the DHA matrix, chlorisondamine and their complexes are consistently lower in the 2-D plot than the ion peaks in the peptide trend line. We designated a separate "aromatic" trend line for these molecular ions with a single benzene ring. Additionally, chlorisondamine fragments occurring from the loss of a quaternary amine or the loss of chlorines are also present along the aromatic trend line.

The ability to separate different classes of compounds

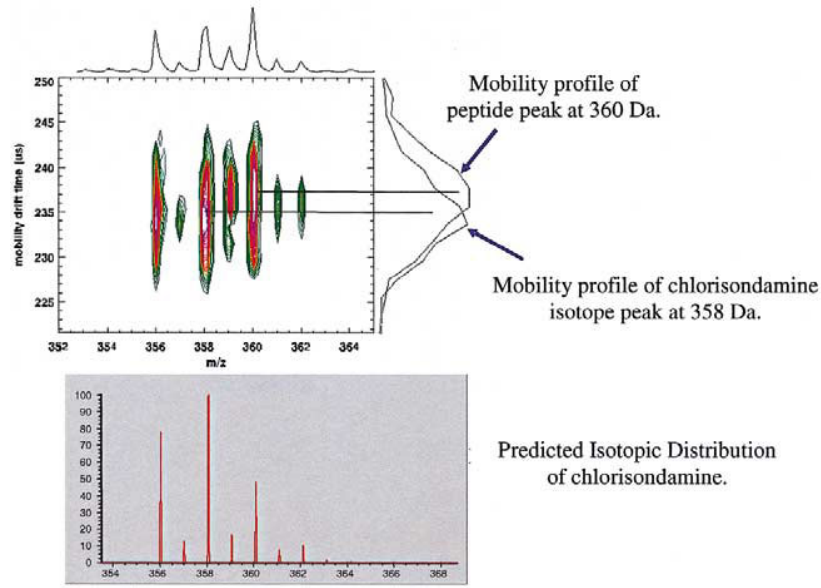

Figure 5. Comparison of mobility-mass region of chlorisondamine $^{\circ}$ from $^{\circ}$ Figure $^{\circ} 4^{\circ}$ with $^{\circ}$ the ${ }^{\circ}$ predicted $^{\circ}$ isotopic ${ }^{\circ}$ distribution $^{\circ}$ of chlorisondamine.

by ion-mobility prior to mass separation aids in the identification of analytes in the low-mass region. This is demonstrated in the identification of the chlorisondamine molecular ion at $358 \mathrm{Da}$ from a native peptide at $360 \mathrm{Da}$, in which the peptide is isobaric with some of the chlorisondamine isotopic peaks. The peptide mass peak at 360 Da was observed in blank tissue with no chlorisondamine present..$^{\circ}$ Figure ${ }^{\circ} 5^{\circ}$ illustrates $^{\circ} \mathrm{a}^{\circ}$ zoomed $^{\circ}$ in $^{\circ}$ view $^{\circ}$ of ${ }^{\circ}$ the chlorisondamine $e^{\circ}$ mobility-mass ${ }^{\circ}$ region ${ }^{\circ}$ from ${ }^{\circ}$ Figure $^{\circ} 4 \mathrm{~b}^{\circ}$ to which the predicted isotopic distribution of chlorisondamine has been appended. Notice how, despite the overlap of the peptide mass peak with the chlorisondamine isotopic peaks, the mobility profile centroid of mass $360 \mathrm{Da}$ is significantly shifted toward longer mobility times compared with the pure chlorisondamine isotope at $358 \mathrm{Da}$. These results show the potential of combined isotopic resolution and mobility separation as a powerful approach for drug identification, which may reduce the need for more complicated techniques (e.g., MS/MS) for unambiguously determining the presence of drug molecules in tissue.

\section{Conclusions}

Lipids are mostly thought of as biomolecules found in membranes and other compartmentalizing or protective layers in the cell. The fact that many therapeutic compounds interact with the lipid bi-layer, which acts as a storage area for such bioactive molecules, is often ignored. In situ analysis of phospholipids from rat brain tissue sections was performed using both MALDITOFMS and MALDI-IM-TOFMS. Molecular species of phosphatidylcholine, phospatidylethanolamine, and sphingomyelin, three major classes of phospholipids were detected by accurately determining their parent ion mass and by corroborating their identity as lipid on the basis of their specific ion mobility. Mass spectra were dominated by peaks associated with the phospholipid molecular species PC 32:0, PC 34:1, and SM 18:0. 
Noncovalent complexes between chlorisondamine and phospholipids were detected in rat brain tissue slices with chlorisondamine added directly. Direct tissue analysis with MALDI-IM-TOFMS produced 2-D contour plots of ion mobility time versus $\mathrm{m} / \mathrm{z}$ with separate trend lines corresponding to lipids, peptides, drug/ matrix (aromatics), and oligonucleotides. The separation of chlorisondamine from native biomolecules along the aromatic trend line allowed for the identification of chlorisondamine and its fragments. Future studies will focus on mapping of phospholipid species in brain and other tissues. Additional studies will include the detection and mapping of chlorisondamine and other drugs in tissue from specimens administered to the drug of interest. In particular, MALDI-IM-TOFMS will be used because of its ability to separate molecules by class, which should make the identification of low-molecular weight drug molecules easier when compared to conventional MALDI-TOFMS.

\section{Acknowledgments}

JAS thanks Marjorie Schultz for the personal funds used to support Ionwerks' participation in this study. Ionwerks is also grateful for partial support from NIDA phase I SBIR contract N43DA-2-7727. ASW thanks Drs. Roy Wise and Barry Hoffer for intellectual support, ONDCP for instruments funding, without which this and other projects could not have been done, and Mr. Brian Schofield of Johns Hopkins University, Bloomberg School of Public Health for the discussion and suggestions in cresyl violet staining and cryosection technique.

\section{References}

1. Cuzner, M. L.; Davison, A. N.; Gregson, N. A. The Chemical Composition of Vertebrate Myelin and Microsomes. J. Neurochem. 1965, 12, 469-481.

2. Fujiwaki, T.; Yamaguchi, S.; Sukegawa, K.; Taketomi, T. Application of Delayed Extraction Matrix-Assisted Laser Desorption Ionization Time-of-Flight Mass Spectrometry for Analysis of Sphingolipids in Tissues from Sphingolipidosis Patients. J. Chromatogr. B 1999, 731, 45-52.

3. He, X.; Chen, F.; McGovern, M. M.; Schuchman, E. H. A Fluorescence-Based, High-Throughput Sphingomyelin Assay for the Analysis of Niemann-Pick Disease and Other Disorders of Sphingomyelin Metabolism. Anal. Biochem. 2002, 306, 115-123.

4. Han, X.; Holtzman, D. M.; McKeel, D. W., Jr.; Kelley, J.; Morris, J. C. Substantial Sulfatide Deficiency and Ceramide Elevation in Very Early Alzheimer's Disease: Potential Role in Disease Pathogenesis. J. Neurochem. 2002, 82, 809-818.

5. Murphy, E. J.; Schapiro, M. B.; Rapoport, S. I.; Shetty, H. U. Phospholipid Composition and Levels are Altered in Down Syndrome Brain. Brain Res. 2000, 867, 9-18.

6. Woods, A. S.; Moyer, S. C.; Wang, H-Y. J.; Wise, R. A. Interaction of Chlorisondamine with the Neuronal Nicotinic Acetylcholine Receptor. J. Proteome Res. 2003, 2, 207-212.

7. Woods, A. S.; Ugarov, M.; Egan, T.; Koomen, J.; Gillig, K. J.; Fuhrer, K.; Gonin, M.; Schultz, J. A. Lipid/Peptide/Nucleotide Separation with MALDI-Ion Mobility-TOF MS. Anal. Chem. 2004, 76, 2187-2195.

8. Woods, A. S. The Mighty Arginine, the Stable Quaternary Amines, the Powerful Aromatics, and the Aggressive Phosphate: Their Role in the Noncovalent Minuet. J. Proteome Res. 2004, 3, 478-484.
9. Murphy, R. C. Mass Spectrometry of Phospholipids; Illuminati: Denver, 2002; Chap II.

10. Schwartz, S. A.; Reyzer, M. L.; Caprioli, R. M. Direct Tissue Analysis Using Matrix-Assisted Laser Desorption/Ionization Mass Spectrometry: Practical Aspects of Sample Preparation. J. Mass Spectrom. 2003, 38, 699-708.

11. Chaurand, P.; Caprioli, R. M. Direct Profiling and Imaging of Peptides and Proteins from Mammalian Cells and Tissue Sections by Mass Spectrometry. Electrophoresis 2002, 23, 31253135.

12. Todd, P. J.; Schaaff, T. G.; Chaurand, P.; Caprioli, R. M. Organic Ion Imaging of Biological Tissue with Secondary Ion Mass Spectrometry and Matrix-Assisted Laser Desorption/ Ionization. J. Mass Spectrom. 2001, 36, 355-369.

13. Rujoi, M.; Estrada, R.; Yappert, M. C. In Situ MALDI-TOFMS Regional Analysis of Neutral Phospholipids in Lens Tissue. Anal. Chem. 2004, 76, 1657-1663.

14. Baumbach, J. I.; Eiceman, G. A. Ion Mobility Spectrometry: Arriving on Site and Moving Beyond a Low Profile. Appl. Spectrosc. 1999, 53, 338A-355A.

15. McDaniel, E. W; Mason, E. A. The Mobility and Diffusion of Ions in Gases; Wiley: New York, 1973; pp 68-72

16. Collins, D. C.; Lee, M. L. Developments in Ion Mobility Spectrometry-Mass Spectrometry. Anal. Bioanal. Chem. 2002, $372,66-73$.

17. Clemmer, D. E.; Jarrold, M. F. Ion Mobility Measurements and their Applications to Clusters and Biomolecules. J. Mass Spectrom. 1997, 32, 577-592.

18. Kim, S. H.; Spangler, G. E. Ion Mobility Spectrometry of Two Structurally Different Ions Having Identical Ion Mass. Anal. Chem. 1985, 57, 567-569.

19. Gillig, K. J.; Ruotolo, B.; Stone, E. G.; Russell, D. H.; Fuhrer, K.; Gonin, M.; Schultz, A. J. Coupling High-Pressure MALDI with Ion Mobility/Orthogonal Time-of-Flight Mass Spectrometry. Anal. Chem. 2000, 72, 3965-3971.

20. Von Helden, G.; Wyttenbach, T.; Bowers, M. T. Conformation of Macromolecules in the Gas Phase: Use of Matrix-Assisted Laser Desorption Methods in Ion Chromatography. Science 1995, 267, 1483-1485.

21. Paxinos, G.; Watson, C. The Rat Brain in Stereotaxic Coordinates; Academic: 1997.

22. Barenholz, Y.; Thompson, T. E. Sphingomyelin: Biophysical Aspects. Chem. Phys. Lipids 1999, 102, 29-34.

23. Petkovic, M.; Schiller, J.; Muller, M.; Benard, S.; Reichl, S.; Arnold, K.; Arnhold, J. Detection of Individual Phospholipids in Lipid Mixtures by Matrix-Assisted Laser Desorption/Ionization Timeof-Flight Mass Spectrometry: Phosphatidylcholine Prevents the Detection of Further Species. Anal. Biochem. 2001, 289, 202-216.

24. Estrada, R.; Yappert, M. C. Alternative Approaches for the Detection of Various Phospholipid Classes by Matrix-Assisted Laser Desorption/Ionization Time-of-Flight Mass Spectrometry. J. Mass Spectrom. 2004, 39, 412-422.

25. Byrdwell, W. C. Dual Parallel Mass Spectrometers for Analysis of Sphingolipid, Glycerophospholipid, and Plasmalogen Molecular Species. Rapid Commun. Mass Spectrom. 1998, 12, 256-272.

26. Wu, Y.; Wang, J.; Sui, S-F. Characterization of Phospholipids by Electron Impact, Field Desorption, and Liquid Secondary Ion Mass Spectrometry. J. Mass Spectrom. 1997, 32, 616-625.

27. Woods, A. S.; Huestis, M. A. A Study of Peptide-Peptide Interaction by Matrix-Assisted Laser Desorption/Ionization. J. Am. Soc. Mass Spectrom. 2001, 12, 88-96.

28. Ruotolo, B. T.; Gillig, K. J.; Stone, E. G.; Russell, D. H. Peak Capacity of Ion Mobility Mass Spectrometry: Separation of Peptides in Helium Buffer Gas. J. Chromatogr. B 2002, 782, 385-392. 